\title{
Incidence of bone formation by whole bone marrow cell suspensions and by its stromal cell cultures injected into kidney parenchyma of Balb/c mice
}

\author{
K. Włodarski ${ }^{1}$, A. Wojtowicz ${ }^{2}$, A. Brodzikowska ${ }^{3}$, R. Galus ${ }^{1}$ \\ ${ }^{1}$ Chair and Department of Histology and Embryology, Medical University of Warsaw, Poland \\ ${ }^{2}$ Department of Oral Surgery, Medical University of Warsaw, Poland \\ ${ }^{3}$ Department of Preventive Stomatology, Medical University of Warsaw, Poland
}

[Received 7 May 2014; Accepted 29 May 2014]

\begin{abstract}
The evaluation of incidence of bone formation by whole syngeneic bone marrow cell suspension and by bone marrow stromal cell cultured in vitro injection into kidney parenchyma was done. Bone tissue was found in 26 kidneys out of 100 injected with whole bone marrow cells suspension. Cultured stromal bone marrow cells grafted into kidney parenchyma produced ossicles in only 4 out of 101 injected kidneys. Such low incidence of bone forming ability of the marrow stromal cell cultures grafted into kidney indicate their useless for study on bone histogenesis in the kidney by murine marrow stromal cell cultures. (Folia Morphol 2014; 73, 4: 482-485)
\end{abstract}

Key words: kidney, mice, osteogenesis

\section{INTRODUCTION}

In addition to haematopoietic cells which differentiate into blood cells, bone marrow contains a stromal component, the complex of cells which provide the environment (a niche) for maintenance and differentiation of blood cell precursors.

Haematopoietic cells derive from the so called haematopoietic stem cell, a multipotent cell able to proliferate and differentiate into all types of blood circulating cells.

Stromal cells are derived from mesenchymal stem cells (MSC), which develop into a range of mesenchymal cells - fibroblasts, osteoblasts, adipocytes, chondrocytes and endothelial cells.

Although the interplay between the haematopoietic and stromal cells is well established $[7,15,16]$, until recently no transition of cells from the haematopoietic compartment into cells belonging to the stromal compartment were reported. More recent data, however, indicates the possibility of generation of some stromal cells, such as osteoblasts and adipocytes, from the haematopoietic compartment [17]. Thus, a new hypothesis postulating a common precursor cell for both haematopoietic and stromal compartments emerged $[2,5,7,17]$.

MSC were first isolated from bone marrow stromal cultures by Friedenstein (1976) [3], who described them as "mechanocytes". These cells are able to differentiate both in vivo and in vitro into osteogenic cells, thus are considered as osteoblast precursors and are widely used as osteogenic cells for tissue engineering purposes and for bone repair/healing. The bone marrow itself, as a rich source of osteoprogenitors, or isolated bone marrow-derived stromal cells propagated in vitro are both used as a source of osteogenic cells for bone reconstruction $[1,8,11]$.

Address for correspondence: Dr Ryszard Galus, Chair and Department of Histology and Embryology, Medical University of Warsaw, ul. Chałubińskiego 5, 02-004 Warszawa, Poland, tel: +48 226281041 ext. 1418; fax: +48 2262952 82, e-mail: rkgalus@wp.pl 
In this communication paper a comparison of bone formation in the mouse kidneys by syngeneic whole bone marrow cell suspension and by a suspension of bone cultured marrow stromal cells was undertaken.

\section{MATERIALS AND METHODS}

\section{Animals and treatment}

Three-month old female syngeneic Balb/c mice weighing 25-30 g, bread in the animal room of the Chair and Department of Histology and Embryology of our University, were used in accordance with the Medical University of Warsaw guidelines for the care and use of laboratory animals (permission no. 54 of Ethical Committee granted to Dr Ryszard Galus). Institutional guidelines for the care and treatment of laboratory animals were observed. The mice were kept up to 8 per cage with free access to mouse chow and water. For surgery mice were anaesthetised with an intraperitoneal injection of $0.15 \mathrm{~mL}$ of $0.36 \%$ chlorohydrate. Animals were sacrificed by cervical dislocation.

\section{Collection of bone marrow cells}

Bone marrow cells were flushed out from femoral cavities by syringing with minimal essential medium (MEM, Gibco, UK). Pooled marrow from several animals was centrifuged and the cell suspension was used either immediately for transplantation into the kidneys or for stromal cell culture for later use.

For freshly-harvested marrow cells, the contents of 2 femoral cavities (ca $10 \times 10^{6}$ of nucleated cells) were suspended in $0.5 \mathrm{~mL}$ of MEM and injected in $0.1-0.15 \mathrm{~mL}$ into the surgically exposed right kidney of experimental mice, each mice therefore receiving $2-3 \times 10^{6}$ cells.

\section{Stromal cell culture}

The bone marrow cell suspension was cultured in Falcon plastic flasks (Corning Life Sciences, USA) in the MEM supplemented with foetal calf serum and antibiotics (Penicillin/Streptomycin, PAA laboratories $\mathrm{GmbH}$, Austria). Two-four days later the medium was replenished and flask-adherent stromal cells were cultured for a further 8 to 30 days. Cells were trypsinised with $0.25 \%$ trypsin solution (Gibco, Scotland) and detached with a rubber scraper, washed in phosphate-buffered saline and ca 1 million of cells were injected into surgically exposed kidney. The viability of cells was ca $80 \%$, as estimated by the trypan blue exclusion test.

The efficiency of the graft cell response was determined by the presence of bone in the kidney examined histologically. For the analysis paraffin-embedded $10 \mu \mathrm{m}$ serial sections were stained with haematoxylin and eosin.

\section{Statistical analysis}

SPSS 19 software (IBM SPSS Statistics, Armonk, NY, USA) was used for statistical data processing. The comparison of the incidence of bone formation by whole bone marrow and by stromal cells grafting was evaluated by analysis of $95 \%$ confidence interval (Cl).

\section{RESULTS}

The numbers of grafts performed and the incidence of bone formation following short term (8-10 days) and long-term (15-30 days) stromal culture administration are presented in the Table 1.

Administration of whole bone marrow cell suspension into kidney parenchyma of syngeneic recipient mice as evaluated $10-15$ days post grafting or 16-30 days post grafting gave similar results. The incidence

Table 1. The incidence of bone formation in the kidney parenchyma following injection of syngeneic whole bone marrow cell suspension and of cultured bone marrow-derived stromal cells

\begin{tabular}{lccc}
\hline Injected cells & $\begin{array}{c}\text { Duration of experiment } \\
\text { [days] }\end{array}$ & $\begin{array}{c}\text { No. of kidneys } \\
\text { injected }\end{array}$ & $\begin{array}{c}\text { No. of kidneys with bone } \\
\text { ossicles (\% of bone incidence) }\end{array}$ \\
\hline Whole bone marrow cell suspension & $10-15$ & 27 & $6(22.2 \%)$ \\
& $16-30$ & 71 & $18(25.4 \%)$ \\
Total & & 100 & $26(26 \%)^{*}$ \\
Stromal cells cultured for: & $11-20$ & & $4(4.8 \%)$ \\
$8-10$ days & $11-20$ & 83 & $0(0 \%)$ \\
$11-20$ days & & 18 & $4(4 \%)^{*}$ \\
Total & & 101 & \\
\hline
\end{tabular}

${ }^{*}$ Statistically significant difference of bone incidence at $95 \%$ confidence interval 


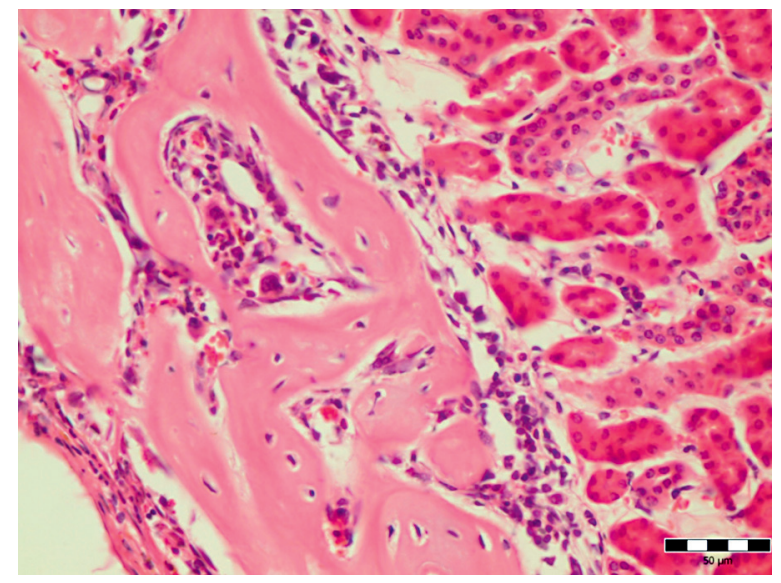

Figure 1. Bone formation in the kidney following subcapsular injection of syngeneic whole bone marrow cell suspension; haematoxylin-eosin staining, scale bar: $50 \mu \mathrm{m}$.

of bone formation was lower than expected, reaching $25 \%$ of cases (Table 1 ). In all instances bone trabeculae were formed by intramembranous ossification, without any cartilage formation. Bone trabeculae were usually covered by osteoblasts, but bone marrow was observed inside ectopic bone only in 3 out of 18 cases of older bone (Fig. 1)

Surprisingly, injection of cultured stromal cells resulted in a markedly lower incidence of bone formation than followed administration of whole marrow cell suspension. In only 4 out of 101 implanted mice was the evidence of renal osteogenesis. Furthermore, osteogenesis occurred exclusively following injection of cells from short-term (5-8 days) stromal cultures. In a group of stromal cells cultured longer, for 15-20 days, no bone formation was observed (Table 1).

The differences of bone incidence between whole bone marrow vs. stromal cells was statistically significant at $95 \%$ of $\mathrm{Cl}$ (incidence $0.26,95 \% \mathrm{Cl} 0.174-0.346$ vs. incidence $0.04 ; 95 \% \mathrm{Cl} 0.001-0.078$ ) (Fig. 1).

\section{DISCUSSION}

The incidence of formation of bone following implantation of whole bone marrow cell suspension into kidney parenchyma by the technique used here was rather low. The reason for it could be leaking of grafted cells from the kidney during manipulation (insertion) of exposed kidney to locate it back into abdominal cavity or during presence there. The number of injected cells exceeded the minimum known to initiate osteogenesis in the muscles $[9,14]$.
One can consider that the extent of bone in heterotopic transplants of marrow stromal cells increases with increased mesenchymal stem cell number, and a threshold number of MSC is necessary for bone formation. A high local threshold density of bone marrow stromal cells is needed for formation of bone [9]. The need of sufficient bone marrow concentration to trigger bone formation was earlier reported also by Tavassoli et al. (1971) [14].

The protocol of MSC cultures applied in our experiment could, at least in part, explain the low incidence of bone formation. We have cultured stromal cells in standard medium on plastic. Expansion of MSC on conventional tissue culture plastic attenuates in vitro osteogenic differentiation and in vivo osteogenesis. To maintain osteogenic differentiation potential, the MSC should be propagated on collagen matrix [10].

Another reason for a poor osteogenic capability of marrow stromal cells grafted into the kidney could be the lack of added osteogenic differentiation factors to the culture medium, such as PTH, TGF-B, $B M P$, vitamin $D$. Such conditioning distinct increases differentiation $[4,12,13]$.

In contrast to low bone incidence by syngeneic bone marrow stromal cell cultures, Krebsbach et al. (1997) [6] reported consistently formation of capsule of cortical-like bone surrounding a cavity with active haematopoiesis in immunodeficient mice following subcutaneous transplantation of marrow stromal fibroblast loaded into gelatin, polyvinyl sponges and collagen matrices. Likely the procedure applied by them prevented the loss of grafted cells from implant bed [6].

Nevertheless, the primary bone marrow stromal cell culture was greatly inferior to freshly-isolated whole bone marrow in osteoinduction. Whole bone marrow cell suspension on introduction into kidney parenchyma resulted with bone formation in only $25 \%$ of mice, a value less than expected, and as was reported by others on subcutaneously implanted bone marrow cells [6].

The technique of bone marrow stromal cell culture applied in this experiment was simple and the tissue culture medium was not fortified by osteotropic agents.

To our knowledge, this is the first report on osteogenesis incidence by freshly-isolated bone marrow cells and by marrow stromal cell cultures in the mouse kidney. 
The results presented in this paper indicate for a limited utility of intrarenal administration of presumably osteogenic cells for the examination of their bone-forming capability in mice. Low incidence of osteogenesis in the applied model could be in part explained by improper ratio of the volume of cell suspension to the volume of the injected kidney and thus by leaking of grafted cells from kidney, so the critical cell density is not achieved.

\section{CONCLUSIONS}

In mice, the technique of intrarenal administration of syngeneic bone marrow cells for in vivo evaluation of their osteogenic potency is not reliable as the incidence of bone formation in this model is low, nor exceeding $25 \%$.

\section{ACKNOWLEDGEMENTS}

We thank Dr Wynn Parry from the University of Liverpool for critical reading of the manuscript and Mrs. Ewa Wiśniewska for laboratory work.

\section{REFERENCES}

1. Connolly JF (1998) Clinical use of marrow osteoprogenitor cells to stimulate osteogenesis. Clin Orthop Rel Res, 355S: S257-S266.

2. Dominici M, Pritchard C, Garlits JE, Hofman TJ, Persons DA, Horwitz EM (2004) Hematopoietic cells and osteoblasts are derived from a common marrow progenitor after bone marrow transplantation. Proc Natl Acad Sci, 101: 11761-11766.

3. Friedenstein AJ (1976) Precursor cells of mechanocytes. Int Rev Cytol, 47: 327-359.

4. Hayashi O, Katsube Y, Hirose M, Ohgushi H, Ito H (2008) Comparison of osteogenic ability of rat mesenchymal stem cells from bone marrow, periosteum, and adipose tissue. Calcif Tissue Int, 82: 238-247.

5. Itoh S, Aubin JE (2009) A novel purification method for multipotential skeletal stem cells. J Cell Biochem, 108: 369-377.

6. Krebsbach PH, Kuznetsov SA, Satomura K, Emmons RV, Rowe DW, Robey PG (1997) Bone formation in vivo: comparison of osteogenesis by transplanted mouse and human marrow stromal fibroblasts. Transplantation, 63: 1059-1069.

7. Kuznetsov SA, Rimunucci M, Ziran N, Tsutsui TW, Corsi A, Calvi L, Kronenberg HM, Schipani E, Robey PG, Bianco P (2004) The interplay of osteogenesis and hematopoiesis: expression of a constitutively active PTH/PTHrP receptor in osteogenic cells perturbs the establishment of hematopoiesis in bone and of skeletal stem cells in the bone marrow. J Cell Biol, 167: 1113-1122.

8. Maniatis A, Tavassoli M, Crosby WH (1971) Origin of osteogenic precursor cells in extramedullary marrow implants. Blood, 38: 569-575.

9. Mankani MH, Kuznetsov SA, Robey PG (2007) Formation of hematopoietic territories and bone by transplanted human bone marrow stromal cells requires a critical cell density. Exp Hematol, 35: 995-1004.

10. Mauney JR, Kirker-Head C, Abrahamson L, Gronowicz G, Volloch V, Kaplan DL (2006) Matrix-mediated retention of in vitro osteogenic differentiation potential and in vivo bone-forming capacity by human adult bone marrow derived mesenchymal stem cells during in vivo expansion. J Biomed Mater Res, 79: 464-475.

11. Paley D, Young MCh, Wiley AM, Fornasier VL, Jackson RW (1986) Percutaneous bone marrow grafting of fractures and bony defects. An experimental study in rabbits. Clin Orthop Rel Res, 208: 300-312.

12. Prabhakar U, James IE, Dodds RA, Lee-Rykaczewski E, Riemen DJ, Lipshutz D, Trulli S, Jonak Z, Tan KB, Drake FH, Gowen M (1998) A novel human bone marrow stroma-derived cell line TF 274 is highly osteogenic in vitro and in vivo. Calcif Tissue Int, 63: 214-220.

13. Rickard DJ, Kazhdan I, Leboy PS (1995) Importance of 1,25-dihydroxyvitamin $D$ and the nonadherent cells of marrow for osteoblast differentiation from rat marrow stromal cells. Bone, 16: 671-678.

14. Tavassoli M, Maniatis A, Binder RA, Crosby WH (1972) Studies on marrow histogenesis. Growth characteristics of extramedullary marrow autotransplantation. Proc Soc Exp Biol Med, 138: 868-870.

15. Teichman RS, Emerson SG (1994) Human osteoblasts support hematopoiesis through the production of granulocyte colony-stimulating factor. J Exp Med, 179: 1672-1682.

16. Vaughan J (1981) Osteogenesis and haematopoiesis. Lancet, 18: 133-135.

17. Włodarski KH (2011) Haematopoietic and osteogenic bone marrow stem cells. Orthop Traumatol Rehabil, 13: 439-447. 